\author{
Marquette University \\ e-Publications@Marquette
}

2017

\title{
Three Sources of Errors in The Ehrenfest Treatment of Inelastic Scattering and Possible Ways of Resolving Them
}

Alexander Semenov

Marquette University

Dmitri Babikov

Marquette University, dmitri.babikov@marquette.edu

Follow this and additional works at: https://epublications.marquette.edu/chem_fac

Part of the Chemistry Commons

\section{Recommended Citation}

Semenov, Alexander and Babikov, Dmitri, "Three Sources of Errors in The Ehrenfest Treatment of Inelastic Scattering and Possible Ways of Resolving Them" (2017). Chemistry Faculty Research and Publications. 897.

https://epublications.marquette.edu/chem_fac/897 


\title{
Three sources of errors in the Ehrenfest treatment of inelastic scattering and possible ways of resolving them
}

\author{
Alexander Semenov and Dmitri Babikov \\ Chemistry Department, Wehr Chemistry Building, Marquette University, Milwaukee, \\ Wisconsin 53201-1881, USA
}

(Received 5 April 2017; accepted 24 May 2017; published online 12 June 2017)

\begin{abstract}
In order to identify the origin of possible errors in the mixed quantum/classical approach to inelastic scattering [A. Semenov and D. Babikov, J. Chem. Phys. 140, 044306 (2014) and A. Semenov, M.-L. Dubernet, and D. Babikov, J. Chem. Phys. 141, 114304 (2014)], a simplified model is considered that consists of one intermolecular degree of freedom and two intramolecular states, coupled by a simple potential. For this system, analytic derivations are carried out to determine (i) the exact quantum mechanical solution of the inelastic scattering problem, (ii) a simplified version of it with all oscillatory terms neglected, and (iii) the Ehrenfest solution in which the translational motion is described by the mean-field trajectory while the internal molecular motion is treated by the timedependent Schrodinger equation. It is shown that the appropriate choice of velocity for the mean-field trajectory permits to enforce microscopic reversibility and gives results in excellent agreement with full-quantum results. The average velocity method of Billing is rigorously derived as a limiting case (of this more general approach), when reversibility is enforced locally, at the initial moment of time only. It is demonstrated that errors of state-to-state transition probabilities in the Ehrenfest approach occur at lower values of total energy $E$ if the magnitudes of excitation energy $\Delta E$, potential energy difference between the two states $\Delta V$, and coupling of two states $V_{12}$ are large. Possible ways of applying this concept to rotational transitions in real molecules are explored, using examples from CO + CO inelastic scattering. Published by AIP Publishing. [http://dx.doi.org/10.1063/1.4985074]
\end{abstract}

\section{INTRODUCTION}

During the last few years, the Ehrenfest approach for the theoretical treatment of inelastic scattering of molecules has been revisited. ${ }^{1-9}$ Within this approximate method, the timedependent quantum mechanics is used for the description of the internal (rotational and vibrational) states of collision partners, while their relative (translational) motion is described by a classical trajectory, driven by the mean-field potential. ${ }^{10}$ This idea, introduced initially by McCann and Flannery, ${ }^{11,12}$ expanded by Billing and co-workers, ${ }^{13-18}$ and explored by others, ${ }^{19-39}$ has recently bloomed due to the development of the related theory, ${ }^{19-21}$ the demonstration of computational efficiency of the method, ${ }^{6,7,10}$ and a systematic benchmark study of its accuracy. ${ }^{5,10}$ The fully coupled version of this approach, formulated in the body-fixed reference frame and suitable for numerically efficient calculations of any collision partners (including polyatomic molecules ${ }^{5}$ and molecule + molecule systems ${ }^{8,9,40}$, was named as the mixed quantum/classical theory (MQCT). A user-friendly suite of codes is now being packaged and will be made available to the community soon. ${ }^{41}$

So far, MQCT calculations have been reported for $\mathrm{Na}$ $+\mathrm{N}_{2},{ }^{4,5} \mathrm{CO}+\mathrm{He},{ }^{2} \mathrm{H}_{2}+\mathrm{He},{ }^{5} \mathrm{H}_{2} \mathrm{O}+\mathrm{He},{ }^{6} \mathrm{CH}_{3} \mathrm{OOCH}+\mathrm{He},{ }^{7}$ $\mathrm{H}_{2}+\mathrm{N}_{2},{ }^{8} \mathrm{CH}_{3}+\mathrm{He},{ }^{10} \mathrm{H}_{2}+\mathrm{H}_{2},{ }^{9}$ and $\mathrm{H}_{2} \mathrm{O}+\mathrm{H}_{2} \mathrm{O} .{ }^{40}$ Within this set of benchmark systems, one finds light and heavy scattering partners, at high and low collision energies, characterized by differential (over scattering angle) and integral cross sections, mostly for rotational but also for some vibrational transitions, within low-lying and highly excited states, for excitation and quenching but also for the elastic channel. Most of these calculations are in very good and often detailed agreement with the results of the full-quantum method (such as coupledchannel theory), but in few cases non-negligible deviations were reported. ${ }^{10}$ The origin of those deviations, and the reason for excellent accuracy of MQCT in all other cases, remained not entirely clear. Since the Ehrenfest method is approximate, it is reasonable to expect that it may be less accurate in some systems or collision regimes, so, it is particularly important to come out with a set of well-defined criteria for applicability of MQCT.

One may expect that the de Broglie wavelength of collision partners would be one suitable criterion for the accuracy of the mixed quantum/classical method, but we showed that even for the lightest systems (such as $\mathrm{H}_{2}+\mathrm{He}^{5}$ ) and even for the lowest kinetic energies $T$ we studied (on the order of few wavenumbers), the classical approximation is well justified, and MQCT can be rather accurate. For all heavier systems and higher collision energies, one should not worry about the applicability of the classical treatment of the relative motion of atomic or molecular scattering partners.

A more important consideration is the magnitude of (rotational and vibrational) excitation quanta in the molecule, $\Delta E$. One may ask why quantization is a problem in a method where the internal motion is treated with quantum mechanics? Well, the internal (quantized) degrees of freedom are coupled to the external degrees of freedom, treated classically. Since no couplings are neglected within MQCT, the internal quantized energy is exchanged with continuous 
translational energy. A known and related problem of the Ehrenfest approach is the violation of microscopic reversibility. In the past, we showed, by a set of numerical "experiments" on a model system, ${ }^{2}$ that the microscopic reversibility and the accuracy of MQCT are both improved as the size of the quantum, $\Delta E=E_{2}-E_{1}$, is reduced. If the value of $\Delta E$ is significant (e.g., vibrational states), then probabilities of transitions between two states, say $P_{1 \rightarrow 2}$ (excitation) and $P_{2 \rightarrow 1}$ (quenching) computed by running two trajectories with the same total energy $E$, are drastically different, which violates reversibility. This is because transition is determined by the collision velocity, but velocities of these two trajectories are very different, determined by the initial kinetic energy $T_{\infty}=E-E_{1}$ for excitation and by $T_{\infty}=E-E_{2}$ for quenching. One $a d h o c$ solution to this problem was proposed by Billing. ${ }^{13}$ To enforce reversibility, by construct, his prescription was to launch a single trajectory with average velocity (of those two cases), which means that the effective or working kinetic energy $U$ should be such that $\sqrt{U}=\left(\sqrt{E-E_{1}}+\sqrt{E-E_{2}}\right) / 2$. We tested this method in our MQCT calculations, for all systems listed above, and found that it works really well. It becomes particularly important at lower collision energies, near excitation threshold, when the excitation quantum $\Delta E$ represents a significant fraction of the total energy $E$. Without this average velocity trick, the results of MQCT (or any other Ehrenfestbased method) would be inaccurate in the low energy regime, and the problem would be more severe for systems with large quanta.

Interestingly, Billing did not really derive his average velocity method, although in his book ${ }^{13}$ he presented several qualitative arguments in support of the method, by drawing analogies with other similar theories. One goal of our paper is to give a rigorous derivation of the average velocity method using a simple two-level system, where both the exact quantum and the mixed quantum/classical solutions can be derived analytically. In this case, it is easy to see their similarities and differences, and is possible to tell how to force the Ehrenfest trajectory to give results very close to the full-quantum results.

However, our experience with MQCT shows that the average velocity method is not always sufficient. There must be another issue (or issues) with the Ehrenfest approach, besides enforcing reversibility with respect to the size of the quantum $\Delta E$. For example, we saw in the past ${ }^{10}$ that some state-tostate transitions are reproduced rather accurately by MQCT, while for some other transitions in the same molecule, MQCT treatment leads to significant errors (when compared vs. exact full-quantum results). Obviously, since we are talking about transitions within the same molecule, the values of the de Broglie wavelength, the collision energy, and the excitation quanta are either exactly the same or comparable, for all these processes. Thus, there must be another property of the molecule, and/or a component of the theory, that works for some state-to-state transitions but not for others. In this paper, using the analytic two-state model, we show that the origin of this second problem is in the mean-field assumption, intrinsic to the Ehrenfest treatment.

Finally, we propose a fix to the problem, or actually, a modified method that we call the average path, which permits to avoid most of the problems in the standard Ehrenfest approach. We discuss the limits of applicability of this method and test it by applying it to one real system, namely, to the inelastic scattering of $\mathrm{CO}+\mathrm{CO}$, using the potential energy surface from the recent literature. ${ }^{42,43}$ Advantages and disadvantages of this method are outlined, and possible avenues for its development and applications are discussed.

\section{THEORY}

\section{A. The exact quantum framework}

Consider a two-state system described by one internal coordinate $s$ (vibrational, rotational) that scatters off a structure-less collision partner (an atom), and the collision process is described by one (translational) coordinate $x \geq 0$. The total wave function of such system is $\Psi(x, s)=\psi_{1}(x) \varphi_{1}(s)$ $+\psi_{2}(x) \varphi_{2}(s)$, where $\psi_{i}(x)$ describe scattering while $\varphi_{i}(s)$ describe the internal motion. The time-independent Schrodinger equation is $\hat{H} \Psi=E \Psi$, with Hamiltonian operator $\hat{H}=-\frac{\hbar^{2}}{2 m} \frac{d^{2}}{d x^{2}}+\hat{H}_{s}+V(x, s)$, where $\hat{H}_{s}$ is the Hamiltonian of the internal motion with two eigenvalues $E_{1}$ and $E_{2}$, whereas $V(x, s)$ is the remaining part of the potential energy surface of the system (does not have to be separable). We will consider a simple model illustrated by Fig. 1, in which the diagonal matrix elements are infinite at the origin $x=0$, are null outside of the interaction region $(x>a)$, and are constant negative numbers within the interaction region, $0<x \leq a:\left\langle\varphi_{1}(s)|V(x, s)| \varphi_{1}(s)\right\rangle=-V_{11}$ and $\left\langle\varphi_{2}(s)|V(x, s)| \varphi_{2}(s)\right\rangle=-V_{22}$. The off-diagonal (coupling) matrix element is non-zero only in the interaction region, $\left\langle\varphi_{2}(s)|V(x, s)| \varphi_{1}(s)\right\rangle=V_{21}=V_{12}$, and can be either positive or negative. Substituting $\Psi$ into the Schrodinger equation and projecting $\varphi_{1}(s)$ and $\varphi_{2}(s)$ out, one obtains the following system of coupled equations (within the interaction region, 0 $<x \leq a)$ :

$\left\{\begin{array}{l}-\frac{\hbar^{2}}{2 m} \frac{d^{2}}{d x^{2}} \psi_{1}(x)+E_{1} \psi_{1}(x)-V_{11} \psi_{1}(x)+V_{12} \psi_{2}(x)=E \psi_{1}(x) \\ -\frac{\hbar^{2}}{2 m} \frac{d^{2}}{d x^{2}} \psi_{2}(x)+E_{2} \psi_{2}(x)-V_{22} \psi_{2}(x)+V_{12} \psi_{1}(x)=E \psi_{2}(x)\end{array}\right.$

Outside of the interaction region, $x>a$, the wave function is given by (see Fig. 1)

$$
\Psi(x, s)=-e^{-i k_{1} x} \varphi_{1}(s)+C_{1} e^{i k_{1} x} \varphi_{1}(s)+C_{2} e^{i k_{2} x} \varphi_{2}(s),
$$

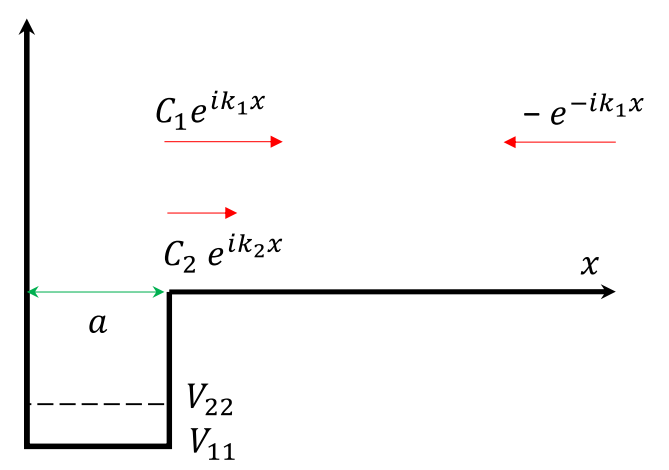

FIG. 1. Schematic of one-dimensional scattering of a two-state system in a simple model problem considered in this work. 
which corresponds to the incoming wave for the initial state 1 and two outgoing waves for the final states 1 and 2, with unknown probability amplitudes $C_{1}$ and $C_{2}$. Signs are chosen such that in the case of elastic scattering $\left(C_{2}=0\right)$, the value of $C_{1}$ is real and positive $\left(C_{1}=1\right)$ and the total wave function $\Psi(x, s)$ turns zero at the origin $x=0$. For the inelastic scattering process $1 \rightarrow 2$, the values of $C_{1}$ and $C_{2}$ should be found by solving system (1) and merging the solution with its asymptotic form (2). Magnitudes of the wave vectors, $k_{1}$ and $k_{1}$, should obey energy conservation, $E=\left(\hbar k_{1}\right)^{2} / 2 m+E_{1}=\left(\hbar k_{2}\right)^{2} / 2 m$ $+E_{2}$, where $E$ is the total energy of the system. The value of the scattering matrix element is given by $S_{12}=\sqrt{k_{2} / k_{1}} C_{2}$. The transition probability is $\left|S_{12}\right|^{2}$, and the corresponding cross section is $\sigma_{12}=\left(\pi / k_{1}^{2}\right)\left|S_{12}\right|^{2}$. Microscopic reversibility is fulfilled by $\left(E-E_{1}\right) \sigma_{12}=\left(E-E_{2}\right) \sigma_{21}$, where $\sigma_{21}=\left(\pi / k_{2}^{2}\right)\left|S_{21}\right|^{2}$ is the cross section for the reverse process and because $S_{21}$ $=S_{12}$ and $\left|S_{12}\right|^{2}=\left|S_{21}\right|^{2}$. Note also that the excitation cross section can be obtained from that for quenching through $\sigma_{12}$ $=\left(k_{2}^{2} / k_{1}^{2}\right) \sigma_{21}$.

One can try to seek the solution of (1) in the following forms: $\psi_{1}(x)=A \sin \kappa x$ and $\psi_{2}(x)=B \sin \kappa x$ (in the range $0<x \leq a$ ). Possible values of the wave vector in the interaction region, $\kappa$ (Greek symbol kappa), are found by substituting these expressions into (1),

$$
\left\{\begin{array}{l}
\frac{\hbar^{2}}{2 m} \kappa^{2} A+E_{1} A-V_{11} A+V_{12} B=E A \\
\frac{\hbar^{2}}{2 m} \kappa^{2} B+E_{2} B-V_{22} B+V_{12} A=E B
\end{array}\right.
$$

and solving for $\kappa$ as follows. One can express $V_{12}$ from each of these equations and multiply one by another. This eliminates coefficients $A$ and $B$, giving a quadratic equation for $\kappa$,

$$
\left(E+V_{11}-\frac{(\hbar \kappa)^{2}}{2 m}-E_{1}\right)\left(E+V_{22}-\frac{(\hbar \kappa)^{2}}{2 m}-E_{2}\right)=V_{12}^{2},
$$

which gives two solutions for $\kappa^{2}$,

$$
\begin{aligned}
\frac{\left(\hbar \kappa_{ \pm}\right)^{2}}{2 m}= & \frac{E+V_{22}-E_{2}+E+V_{11}-E_{1}}{2} \\
& \pm \sqrt{\frac{\left(E_{2}-E_{1}-V_{22}+V_{11}\right)^{2}}{4}+V_{12}^{2}} .
\end{aligned}
$$

We will assume $E>E_{2}$, which makes state 2 energetically accessible, and will only consider $\kappa^{2}>0$ which correspond to the attractive well, as in Fig. 1 (in contrast, the case of $\kappa^{2}<0$ is relevant to the repulsive barrier problem, such as tunneling). Note that in general, kappa depends on both the collision energy $E$ and the properties of the system $\left(E_{1}, E_{2}\right.$, $V_{12}, V_{11}$, and $V_{22}$ ).

Then, from the first of Eq. (3), we obtain

$$
\left(\frac{B}{A}\right)_{ \pm}=\frac{E+V_{11}-\frac{\left(\hbar \kappa_{ \pm}\right)^{2}}{2 m}-E_{1}}{V_{12}}=R \mp \sqrt{R^{2}+1},
$$

where, to characterize the strength of coupling in the system, we introduced the ratio

$$
R=\frac{\Delta E-\Delta V}{2 V_{12}},
$$

with $\Delta E=E_{2}-E_{1}$ and $\Delta V=V_{22}-V_{11}$. Below, it will be convenient to replace the $B$ to $A$ ratio by another parameter, $B / A=p$. So, $p_{ \pm}=R \mp \sqrt{R^{2}+1}$. We see that the product of two possible values gives $p_{+} p_{-}=-1$. Other useful properties are $p_{-}+p_{+}=2 R$ and $p_{-}-p_{+}=2 \sqrt{R^{2}+1}$.

The fact that the $B$ to $A$ ratio can take two possible values means that the most general solution of (1) is $\psi_{1}(x)=A_{+}$ $\sin \kappa_{+} x+A_{-} \sin \kappa_{-} x \quad$ and $\quad \psi_{2}(x)=\mathrm{B}_{+} \sin \kappa_{+} x+\mathrm{B}_{-} \sin \kappa_{-} x$, rather than a simpler version assumed in the sentence preceding Eq. (3). This still turns the total wave function $\Psi$ into zero at $x=0$, while in the interaction region $0<x \leq a$,

$$
\begin{aligned}
\Psi(x, s)= & \left(A_{+} \sin \kappa_{+} x+A_{-} \sin \kappa_{-} x\right) \varphi_{1}(s) \\
& +\left(\mathrm{B}_{+} \sin \kappa_{+} x+\mathrm{B}_{-} \sin \kappa_{-} x\right) \varphi_{2}(s) .
\end{aligned}
$$

Without loss of generality, we can assume $\kappa>0$. On the border of the interaction region, at $x=a$, this solution should match Eq. (2). In the Appendix, it is shown that this is achieved by setting

$$
\begin{aligned}
C_{2}= & -2 e^{-i a\left(k_{1}+k_{2}\right)} i k_{1}\left[\kappa_{+} \cos a \kappa_{+} \sin a \kappa_{-}-\kappa_{-} \cos a \kappa_{-} \sin a \kappa_{+}\right] /\left[p_{+}\left(\kappa_{+} \cos a \kappa_{+}-i k_{2} \sin a \kappa_{+}\right)\left(\kappa_{-} \cos a \kappa_{-}-i k_{1} \sin a \kappa_{-}\right)\right. \\
& \left.\times-p_{-}\left(\kappa_{+} \cos a \kappa_{+}-i k_{1} \sin a \kappa_{+}\right)\left(\kappa_{-} \cos a \kappa_{-}-i k_{2} \sin a \kappa_{-}\right)\right] .
\end{aligned}
$$

Then, for transition probability, we can explicitly write

$$
\begin{aligned}
\left|S_{12}\right|^{2}= & \frac{k_{2}}{k_{1}}\left|C_{2}\right|^{2}=4 k_{1} k_{2}\left[\kappa_{+} \cos a \kappa_{+} \sin a \kappa_{-}-\kappa_{-} \cos a \kappa_{-} \sin a \kappa_{+}\right]^{2} /\left[\left(p_{+}-p_{-}\right)^{2}\left(\kappa_{+} \kappa_{-} \cos a \kappa_{+} \cos a \kappa_{-}-k_{1} k_{2} \sin a \kappa_{+} \sin a \kappa_{-}\right)^{2}\right. \\
& \left.+\left(\left(p_{+} k_{1}-p_{-} k_{2}\right) \kappa_{+} \cos a \kappa_{+} \sin a \kappa_{-}-\left(p_{-} k_{1}-p_{+} k_{2}\right) \kappa_{-} \cos a \kappa_{-} \sin a \kappa_{+}\right)^{2}\right]
\end{aligned}
$$

This somewhat complicated expression is exact. It is used in Sec. III to obtain accurate quantum results for this model system. Properties of this formula are further explored in the Appendix, and it is shown that the following simplified version of it can be obtained (by neglecting all oscillating terms):

$$
\left|S_{12}\right|^{2} \approx \frac{2 k_{1} k_{2}\left(\kappa_{+}+\kappa_{-}\right)^{2}}{k_{1}^{2} k_{2}^{2}+\kappa_{+}^{2} \kappa_{-}^{2}} \cdot \frac{\sin ^{2} a\left(\kappa_{+}-\kappa_{-}\right)}{\left(p_{+}-p_{-}\right)^{2}} .
$$


Furthermore, in the limit of large scattering energies (the classical limit, $E \rightarrow \infty$ ), we obtain

$$
\begin{aligned}
\left|S_{12}\right|^{2} & \approx 4 \frac{\sin ^{2} a\left(\kappa_{+}-\kappa_{-}\right)}{\left(p_{+}-p_{-}\right)^{2}} \\
& \approx \frac{1}{R^{2}+1} \sin ^{2}\left\{\frac{a \sqrt{2 m}}{\hbar} \cdot \frac{V_{12} \sqrt{R^{2}+1}}{\sqrt{E}}\right\} .
\end{aligned}
$$

Deriving this approximate formula, we set $k_{1} \approx k_{2} \approx \kappa_{+} \approx \kappa_{-}$, expressed $\kappa_{+}-\kappa_{-}$through $\kappa_{+}+\kappa_{-}$as shown in the Appendix and assumed $E>>E_{1}-V_{11}$ and $E>>E_{2}-V_{22}$.

\section{B. The mixed quantum/classical framework}

The mixed quantum classical Hamiltonian for this problem is $\hat{H}=P_{x}^{2} / 2 m+\hat{H}_{s}+V(x, s)$, where the internal degree of freedom $s$ is still treated quantum mechanically, but the motion along the scattering coordinate $x$ is now described classically, by introducing classical momentum for this degree of freedom, $P_{x}(t)$. In general, it evolves along the Ehrenfest trajectory: $\partial P_{x} / \partial t=-\partial \tilde{V} / \partial x$, driven by the meanfield potential $\tilde{V}(x)=\langle\Psi(s)|V(x, s)| \Psi(s)\rangle$. Here $\Psi(s)$ is the wave function for the internal motion. Its evolution obeys the time-dependent Schrodinger equation, $\hat{H} \Psi=i \hbar \partial \Psi / \partial t$. Expressing wave function through eigenfunctions of two states with time dependent coefficients, $\Psi(s, t)=a_{1}(t) \varphi_{1}(s)$ $+a_{2}(t) \varphi_{2}(s)$, substituting this expression into the timedependent Schrodinger equation, and projecting out $\varphi_{1}(s)$ and $\varphi_{2}(s)$, we obtain

$$
\left\{\begin{array}{l}
a_{1}(t)\left(E_{1}+V_{11}(x)\right)+a_{2}(t) V_{12}(x)=i \hbar \frac{\partial}{\partial t} a_{1}(t) \\
a_{2}(t)\left(E_{2}+V_{22}(x)\right)+a_{1}(t) V_{21}(x)=i \hbar \frac{\partial}{\partial t} a_{2}(t)
\end{array} .\right.
$$

Since in our model, the potential is zero outside of the interaction region, $0<x \leq a$, we can start collision from $x$ $=a$ at $t=0$. As system moves through the interaction region, let us try searching for solutions of Eq. (13) in the following simplest forms: $a_{1}(t)=A \exp (-i \omega t)$ and $a_{2}(t)=B \exp (-i \omega t)$. Substituting these expressions into Eq. (13) gives

$$
\left\{\begin{array}{l}
A\left(E_{1}-V_{11}\right)+B V_{12}=\hbar \omega A \\
B\left(E_{2}-V_{22}\right)+B V_{12}=\hbar \omega B
\end{array} .\right.
$$

This system of equations should be solved for $\omega$ by expressing $V_{12}$ from each, multiplying one by another to eliminate coefficients $A$ and $B$, and solving the resultant quadratic equation

$\hbar \omega_{ \pm}=\frac{E_{1}-V_{11}+E_{2}-V_{22}}{2} \pm \sqrt{\left(\frac{E_{2}-V_{22}-E_{1}+V_{11}}{2}\right)^{2}+V_{12}^{2}}$.

Note that omega depends on properties of the system only, that is, on $E_{1}, E_{2}, V_{12}, V_{11}$, and $V_{22}$. Using one of the equations (14), we can obtain $(B / A)_{ \pm}=p_{ \pm}=R \mp \sqrt{R^{2}+1}$, exactly equivalent to the quantum equations, (6) and (7). Note that Eq. (15) is also very similar to the quantum equation (5).

Again, the fact that there are two solutions, $\omega_{+}$and $\omega_{-}$, means that the general form of the time-dependent coefficients is

$$
\begin{aligned}
& a_{1}(t)=A_{+} \exp \left(-i \omega_{+} t\right)+A_{-} \exp \left(-i \omega_{-} t\right) \\
& a_{2}(t)=B_{+} \exp \left(-i \omega_{+} t\right)+B_{-} \exp \left(-i \omega_{-} t\right)
\end{aligned}
$$

For simplicity, we will assume that only state 1 is populated initially: $a_{1}(0)=1$ and $a_{2}(0)=0$. This gives us $A_{+}+A_{-}=1$ and $p_{+} A_{+}+p_{-} A_{-}=0$. Solving these two equations gives us $A_{+}$ $=p_{-} /\left(p_{-}-p_{+}\right), A_{-}=-p_{+} /\left(p_{-}-p_{+}\right)$, and $B_{+}=-B_{-}=p_{+} p_{-} /\left(p_{-}\right.$ $\left.-p_{+}\right)=-1 /\left(p_{-}-p_{+}\right)$. Here we used $p_{+} p_{-}=-1$. Remember that the denominator in these formulas is positive, $p_{-}-p_{+}$ $=2 \sqrt{R^{2}+1}$.

Note that the total energy $E$ does not show up anywhere in these equations. It is determined by the asymptotic value of kinetic energy of the system (by its classical momentum): $E=T_{\infty}+E_{1}$, where $T_{\infty}=P_{\infty}^{2} / 2 m$. So, $P_{\infty}=-\sqrt{2 m\left(E-E_{1}\right)}$. When the system enters the interaction region, the value of momentum changes according to

$$
\begin{aligned}
E= & \frac{P_{x}^{2}}{2 m}+\left\langle\Psi(s, t)\left|\hat{H}_{s}+V(x, s)\right| \Psi(s, t)\right\rangle \\
= & \frac{P_{x}^{2}}{2 m}+\left(E_{1}-V_{11}\right)\left|a_{1}(t)\right|^{2}+\left(E_{2}-V_{22}\right)\left|a_{2}(t)\right|^{2} \\
& +V_{12} \cdot 2 \operatorname{Re}\left\{a_{1}(t) a_{2}^{*}(t)\right\} .
\end{aligned}
$$

For the model system depicted in Fig. 1, at $t=0$, as a system, being initially at state 1 , just have fallen into the well at $x=a$, we obviously find $P_{x}(t=0)=-\sqrt{2 m\left(E-E_{1}+V_{11}\right)}$ (i.e., momentum receives a sudden boost, as it should be in this problem with a discontinuous interaction potential). As the system moves through the interaction region, probability amplitudes change, according to Eqs. (16). From (16), we have

$$
\begin{aligned}
\left|a_{1}(t)\right|^{2}= & A_{+}^{2}+A_{-}^{2}+2 A_{+} A_{-} \cos \left(\omega_{+}-\omega_{-}\right) t, \\
\left|a_{2}(t)\right|^{2}= & B_{+}^{2}+B_{-}^{2}+2 B_{+} B_{-} \cos \left(\omega_{+}-\omega_{-}\right) t, \\
\operatorname{Re}\left\{a_{1}(t) a_{2}^{*}(t)\right\}= & A_{+} B_{+}+A_{-} B_{-}+\left(A_{+} B_{-}+A_{-} B_{+}\right) \\
& \times \cos \left(\omega_{+}-\omega_{-}\right) t .
\end{aligned}
$$

The substitution of these expressions into Eq. (17) gives several terms. The time-dependent term, proportional to $\cos \left(\omega_{+}-\omega_{-}\right) t$, happens to turn to null. The time independent term transforms as follows:

$$
\begin{aligned}
\left(A_{+}{ }^{2}\right. & \left.+A_{-}^{2}\right)\left(E_{1}-V_{11}\right)+\left({B_{+}}^{2}+B_{-}^{2}\right)\left(E_{2}-V_{22}\right) \\
& +2\left(A_{+} B_{+}+A_{-} B_{-}\right) V_{12}=\left[\left(E_{1}-V_{11}\right)\left(4 R^{2}+2\right)\right. \\
& \left.+2\left(E_{2}-V_{22}\right)-4 R V_{12}\right] / 4\left(R^{2}+1\right)=E_{1}-V_{11} .
\end{aligned}
$$

So, at any moment of time, we have $E=P_{x}{ }^{2} / 2 m+\left(E_{1}\right.$ $-V_{11}$ ), which means that the value of classical momentum of the system, as it moves through the interaction region, remains constant, equal to $P_{x}= \pm \sqrt{2 m\left(E-E_{1}+V_{11}\right)}$. It is not affected by the value of $V_{12}$, which is somewhat counterintuitive but is a property of the system depicted in Fig. 1. 
The total time a particle spends in the interaction region is $\tau=2 m a / P_{x}$, so, the inelastic transition probability is

$$
\begin{aligned}
P_{1 \rightarrow 2} & =\left|a_{2}(\tau)\right|^{2}=B_{+}{ }^{2}+B_{-}{ }^{2}+2 B_{+} B_{-} \cos \left\{2 m a\left(\omega_{+}-\omega_{-}\right) / P_{x}\right\} \\
& =\frac{2-2 \cos \left\{2 m a\left(\omega_{+}-\omega_{-}\right) / P_{x}\right\}}{\left(p_{-}-p_{+}\right)^{2}} \\
& =\frac{4 \sin ^{2}\left\{m a\left(\omega_{+}-\omega_{-}\right) / P_{x}\right\}}{4\left(R^{2}+1\right)} \\
& =\frac{1}{R^{2}+1} \sin ^{2}\left\{\frac{2 m a}{\hbar P_{x}} V_{12} \sqrt{R^{2}+1}\right\}
\end{aligned}
$$

or, finally

$$
P_{1 \rightarrow 2}=\frac{1}{R^{2}+1} \sin ^{2}\left\{\frac{a \sqrt{2 m}}{\hbar} \cdot \frac{V_{12} \sqrt{R^{2}+1}}{\sqrt{E-E_{1}+V_{11}}}\right\} .
$$

We see that, first of all, the transition probability is zero in the limit of negligible coupling, $V_{12} \rightarrow 0$, just as in the fullquantum case. We also see that in the limit of high scattering energies $E>>E_{1}-V_{11}$, the mixed quantum classical result is equivalent to the full-quantum result, Eq. (12).

\section{Merging results of the two methods}

Note, however, that Eq. (19a) is not symmetric with respect to the initial and final states. Namely, for quenching (transition $2 \rightarrow 1$ ), we would obtain

$$
P_{2 \rightarrow 1}=\frac{1}{R^{2}+1} \sin ^{2}\left\{\frac{a \sqrt{2 m}}{\hbar} \cdot \frac{V_{12} \sqrt{R^{2}+1}}{\sqrt{E-E_{2}+V_{22}}}\right\} .
$$

Only in the limit of high energy, Eqs. (19a) and (19b) give equivalent results, fulfilling reversibility, $P_{2 \rightarrow 1}=P_{1 \rightarrow 2}$. But if the energy is on the order of parameters of the system, $E \sim\left(E_{1}-V_{11}\right) \sim\left(E_{2}-V_{22}\right)$, then Eqs. (19a) and (19b) violate reversibility, $P_{2 \rightarrow 1} \neq P_{1 \rightarrow 2}$, and both deviate from the quantum result of Eq. (12). It is important to understand the origin of this difference. Note that in the calculations of excitation and quenching (at the same total energy $E$ ), the mixed quantum/classical trajectories spend different amount of time in the interaction region, since their momenta are different: $P_{x}= \pm \sqrt{2 m\left(E-E_{1}+V_{11}\right)}$ for excitation and $P_{x}= \pm \sqrt{2 m\left(E-E_{2}+V_{22}\right)}$ for quenching. When these trajectories are launched from the asymptotic region $(x \gg a$, where $V_{11}=V_{22}=0$ ), they also have different initial momenta: $P_{\infty}=-\sqrt{2 m\left(E-E_{1}\right)}$ for excitation and $P_{\infty}=-\sqrt{2 m\left(E-E_{2}\right)}$ for quenching. Thus, these two trajectories run at two different speeds and cannot give the same transition probability. The problem is more severe when energies of the states, $E_{1}$ and $E_{2}$, differ significantly. But this discrepancy is expected to vanish in the high energy limit, when $E>>E_{1}-V_{11}$ and $E>>$ $E_{2}-V_{22}$. These properties of the mixed quantum/classical approach are demonstrated in numerical experiments of Sec. III.

But, one can ask the following question: Is it possible to launch such trajectory that the mixed quantum/classical result would agree with the full-quantum result? For this, we should require that at least the arguments of sine functions in Eq. (18) and in Eq. (11) are made equal: $a\left(\kappa_{+}-\kappa_{-}\right)=m a\left(\omega_{+}-\omega_{-}\right) / P_{x}$, which gives the expression for the wanted velocity: $v_{x}=\left(\omega_{+}-\omega_{-}\right) /\left(\kappa_{+}-\kappa_{-}\right)$. Expressing $\kappa_{+}-\kappa_{-}$through $\kappa_{+}+\kappa_{-}$ as shown in the Appendix, we can re-express this velocity thorough purely quantum parameters of the system: $v_{x}=(\hbar / 2 m)$ $\left(\kappa_{+}+\kappa_{-}\right)$. Using Eqs. (5) and (15), we explicitly obtain

$$
\begin{aligned}
v_{x}= & \frac{1}{2} \sqrt{\frac{1}{m}} \\
& \cdot\left[\sqrt{2 E+V_{22}-E_{2}+V_{11}-E_{1}+(\Delta E-\Delta V) \sqrt{1+R^{-2}}}\right. \\
& \left.+\sqrt{2 E+V_{22}-E_{2}+V_{11}-E_{1}-(\Delta E-\Delta V) \sqrt{1+R^{-2}}}\right],
\end{aligned}
$$

which we will call the effective velocity. This lengthy formula can be drastically simplified in the case of weak coupling, $V_{12}$ $\rightarrow 0$ or $R \rightarrow \infty$,

$$
\tilde{v}=\frac{\sqrt{2\left(E-E_{1}+V_{11}\right) / m}+\sqrt{2\left(E-E_{2}+V_{22}\right) / m}}{2} .
$$

This moiety represents the average of two velocities, those two that would normally occur in two independent calculations of excitation and quenching. If the trajectory is forced to move through the interaction region with velocity $\tilde{v}$, this would permit to obtain the essentially exact quantum result using the mixed quantum/classical method.

Practical implementation of this idea would depend on the initial state. If the initial state is 1 and the mixed quantum/classical calculations are carried out for excitation, the initial kinetic energy $T_{\infty}$ for the trajectory must be chosen such that $m \tilde{v}^{2} / 2=T_{\infty}+V_{11}$. If the initial state is 2 and the mixed quantum/classical calculations are carried out for quenching, the condition would be $m \tilde{v}^{2} / 2=T_{\infty}+V_{22}$. In either case, as the system reaches the interaction region at $x=a$, falls into the well, and accelerates (by $V_{11}$ or by $V_{22}$ depending on the initial state), its kinetic energy becomes equal to $m \tilde{v}^{2} / 2$, which guarantees nearly perfect agreement with full-quantum results. It should be stressed that this approach requires launching trajectories for excitation and quenching with two different initial velocities. Only when they reach the interaction region, their velocities become equal to each other [and to the value required by Eq. (20) or Eq. (21)].

Although this approach is attractive, it becomes clear that the effective velocity is well defined and is straightforward to match only in the case of a simple model considered in this paper (shown in Fig. 1). But, in a general case, when the coordinate-dependence of matrix elements of the interaction potential is continuous [as in real systems with smooth $V_{11}(x)$ and $V_{22}(x)$, such as molecule + atom or molecule + molecule], it seems unfeasible to force any trajectory to follow such a path along which the effective velocity condition would remain rigorously satisfied.

One can, however, try to use Eq. (21) as the initial condition, enforcing average velocity at the initial moment of time, at least, when the system is in the asymptotic region $x \rightarrow \infty$, where $V_{11}=V_{22}=0$. Then, a simplified version of Eq. (21) is

$$
\tilde{v}=\frac{\sqrt{2\left(E-E_{1}\right) / m}+\sqrt{2\left(E-E_{2}\right) / m}}{2},
$$

and the initial kinetic energy is chosen as $T_{\infty}=m \tilde{v}^{2} / 2$, for both excitation and quenching trajectories. This simple 
formula, when used to define the initial condition, is equivalent to the average velocity recipe of Billing. ${ }^{13}$ One can also hope that if the potential energy of interaction is small compared to the kinetic energy, $E-E_{1}>>V_{11}(x)$ and $E-E_{2}$ $>V_{22}(x)$, then this condition would remain approximately satisfied during the course of collision, helping to improve reversibility and better agreement with the exact full-quantum result.

It should be stressed that our approach is, in fact, an antithesis of the method of Billing, at least in the nontrivial case of significant $\Delta E$ and $\Delta V$. Indeed, our approach is to match the speeds of excitation and quenching trajectories in the interaction region (which requires launching them with different initial speeds), while the approach of Billing is to make the speeds equal at the initial moment of time (which unavoidably leads to different speeds in the interaction region). Accuracy of one or the other approach may depend on the properties of the system and should be checked by calculations.

\section{NUMERICAL RESULTS FOR MODEL SYSTEM}

In this section, we will compare the results of the mixed quantum/classical theory against the full-quantum results, for several choices of parameters of the model system. Four versions of the mixed quantum/classical theory will be used: a straightforward Ehrenfest approach without any adjustments to enforce reversibility, the average velocity method of Billing based on the simplest equation (22), and two modified versions with a more accurate formula for the effective velocity, those of Eqs. (21) and (20). Two versions of the full-quantum results will be shown: those obtained using the exact formula of Eq. (10) and a simplified formula of Eq. (11) where all oscillatory terms are neglected.

The first set of model parameters is chosen such that $\Delta E, \Delta V$, and $V_{12}$ are all small. The range of parameters is typical to rotationally inelastic scattering of $\mathrm{CO}+\mathrm{CO}$, namely,

$$
\begin{gathered}
m=14 \mathrm{amu}, \quad a=3.1 \mathrm{Bohr}, \quad E_{1}=0, \quad E_{2}=10 \mathrm{~cm}^{-1}, \\
V_{11}=200 \mathrm{~cm}^{-1}, \quad V_{22}=200 \mathrm{~cm}^{-1}, \quad \text { and } \quad V_{12}=60 \mathrm{~cm}^{-1},
\end{gathered}
$$
which we will call system 0 . For this case, the transition probability is plotted in Fig. 2(a) as a function of total energy in the range $100 \leqslant E \leqslant 10000 \mathrm{~cm}^{-1}$. Full-quantum results are shown by black lines: solid for the exact solution, Eq. (10), and dashed for the approximate solution, Eq. (11). We see, first of all, that in the high energy limit, where the dependence is smooth, the approximate quantum formula gives perfect agreement with the accurate quantum formula. At lower energy, the accurate quantum solution shows oscillations of the transition probability. Importantly, the approximate quantum solution goes right through the middle of those oscillations, providing a meaningful representative answer, on average. At very low energies, the deviation of the approximate quantum solution from the exact quantum solution becomes noticeable [see Fig. 2(a)].

Red and blue lines in Fig. 2(a) correspond to the mixed quantum/classical results obtained with two different initial
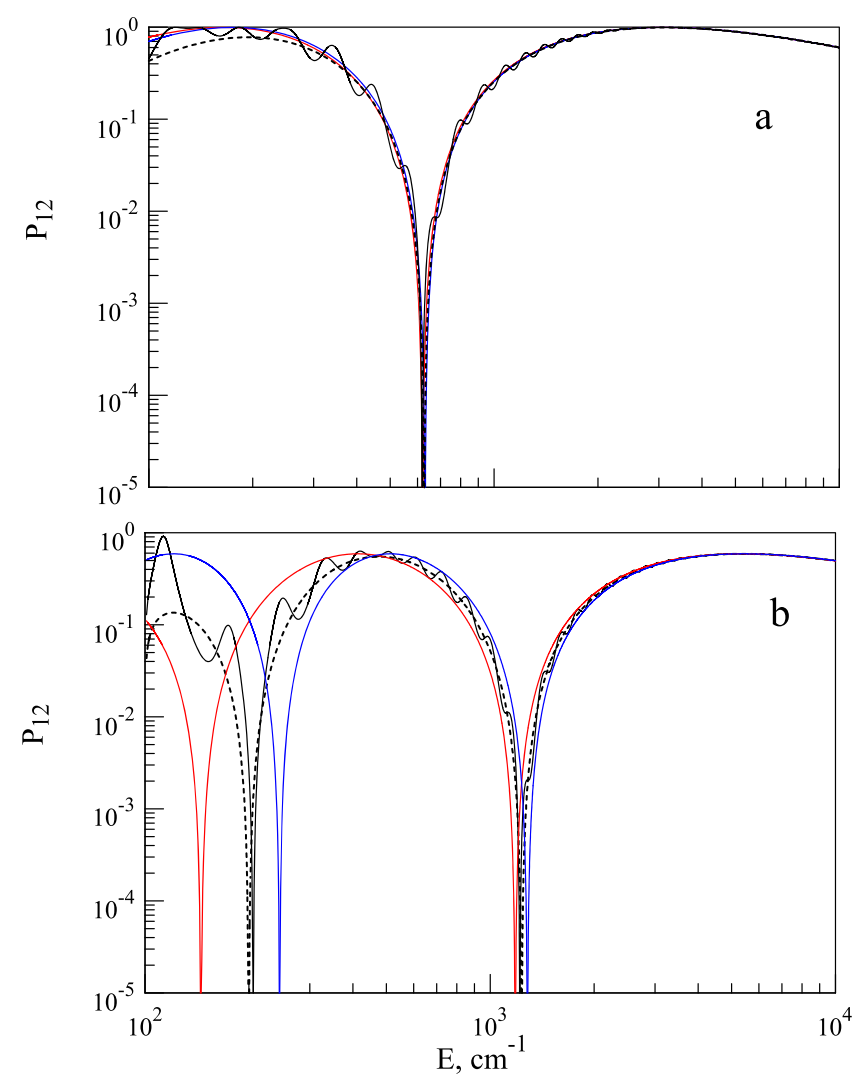

FIG. 2. Transition probability as a function of total energy determined by the straightforward application of the mixed quantum/classical approach for excitation and quenching (red and blue lines, respectively). Results of the exact and approximate full-quantum formula (solid and dashed lines, respectively) are shown for comparison. Upper frame demonstrates excellent agreement for system 0 with a small quantum of internal energy. Lower frame demonstrates poor agreement for system 1 with a large quantum of internal energy.

states: red for the initial state 1 and blue for the initial state 2 . In those two cases, the initial kinetic energies of collision are different (slightly), determined simply by the total energy, and the velocities are different: $v_{1}=\sqrt{2\left(E-E_{1}\right) / m}$ for excitation and $v_{2}=\sqrt{2\left(E-E_{2}\right) / m}$ for quenching. Equations (19a) and (19b) are used to compute the transition probability. We see that the two mixed quantum/classical results agree with each other rather well and are also in excellent agreement with fullquantum results. They do not oscillate (similar to the approximate quantum dependence) and, on average, are even slightly more accurate at low energies than the approximate quantum dependence. The "anti-resonance" behavior at the intermediate energies and the asymptotic behavior at high energies are all perfectly reproduced by the mixed quantum/classical method [see Fig. 2(a)].

From this example, it becomes clear that there may be molecules and collision conditions where the Ehrenfest approach is acceptably accurate without any adjustments. In these cases, calculations can be carried out as excitation or as quenching, but results would be very similar because the difference between $E_{1}$ and $E_{2}$ is small, leading to similar kinetic energies and collision velocities, $v_{1}$ $\approx v_{2}$. This simple conclusion might be quite useful for the description of rotational excitation in polyatomic molecules, 
where the rotational quanta are typically small (e.g., on the order of $0.3 \mathrm{~cm}^{-1}$ in methyl formate, $\mathrm{CH}_{3} \mathrm{OOCH}^{7}$ ). In such cases, reversibility is not an issue. The simplest version of the mixed quantum/classical theory can be employed and is expected to give results in good agreement with full-quantum results.

Now let us increase the value of the internal energy quantum in the system by shifting the second state to $E_{2}=100 \mathrm{~cm}^{-1}$ and keeping all other parameters same as before (call this system 1). In this case, $\Delta E$ is rather large, the same order of magnitude as $V_{11}$ and $V_{22}$. Results of the full-quantum and the mixed quantum/classical methods are presented in Fig. 2(b). In this system, we see two "anti-resonances" in the considered energy range. We also see that the amplitude of quantum oscillations is an order of magnitude larger [compared to system 0, Fig. 2(a)], but the approximate quantum formula still does a good job, on average (dashed vs. solid black lines). However, two results of the mixed quantum/classical method (red and blue) deviate significantly from each other and in opposite directions from the quantum result. The high energy limit is still reproduced perfectly, and the two "antiresonances" are still captured, but the low-energy behavior is rather inaccurate. For example, while in the intermediate energy range, the full-quantum result oscillates between the two mixed quantum/classical results (black line oscillates between red and blue lines), and those two can even be used as a guidance for predicting the range of quantum oscillations, at low energies, the deviations are so significant that discrepancies by several orders of magnitude may occur [see Fig. 2(b)].

The explanation for this behavior is that now, when the values of $E_{1}$ and $E_{2}$ are substantially different, the values of kinetic energies and initial velocities in the mixed quantum/classical calculations of excitation and quenching are also substantially different, in particular when the total energy $E$ is small: $v_{1}=\sqrt{2\left(E-E_{1}\right) / m}$ vs $v_{2}=\sqrt{2\left(E-E_{2}\right) / m}$. Setting up the initial condition using the average velocity of Billing, $\tilde{v}=\left(v_{1}+v_{2}\right) / 2$, helps in this case, as one can see from Fig. 3(a), where we used Eq. (22) to start excitation and quenching trajectories in system 1 . Now the blue and red curves completely coincide [thus, only one can be seen in Fig. 3(a)] and both agree well with the exact full-quantum result. In fact, at low energies, the mixed quantum/classical method (when used properly, here with Billing initial conditions) again gives a slightly better description than the approximate quantum formula of Eq. (11). [As a technical note, we want to mention that in order to implement the approach of Billing, we simply used Eq. (18) with the appropriate value of momentum; we did not really propagate trajectories, since the solution is analytic for this system.]

In our past experience with MQCT, we, indeed, saw situations when the straight application of the Ehrenfest mean-field approach was terribly inaccurate, but a simple correction of average velocity, according to Billing, would improve the result dramatically. Those cases correspond to large internal quanta $\Delta E$, such as vibrational quenching of $\mathrm{CO}(v=1)^{2}$ or rotational quenching of light quantum rotors such as $\mathrm{H}_{2}, 5$ and most transitions in $\mathrm{H}_{2} \mathrm{O}$. In these molecules, reversibility is enforced almost perfectly (by the average velocity choice that
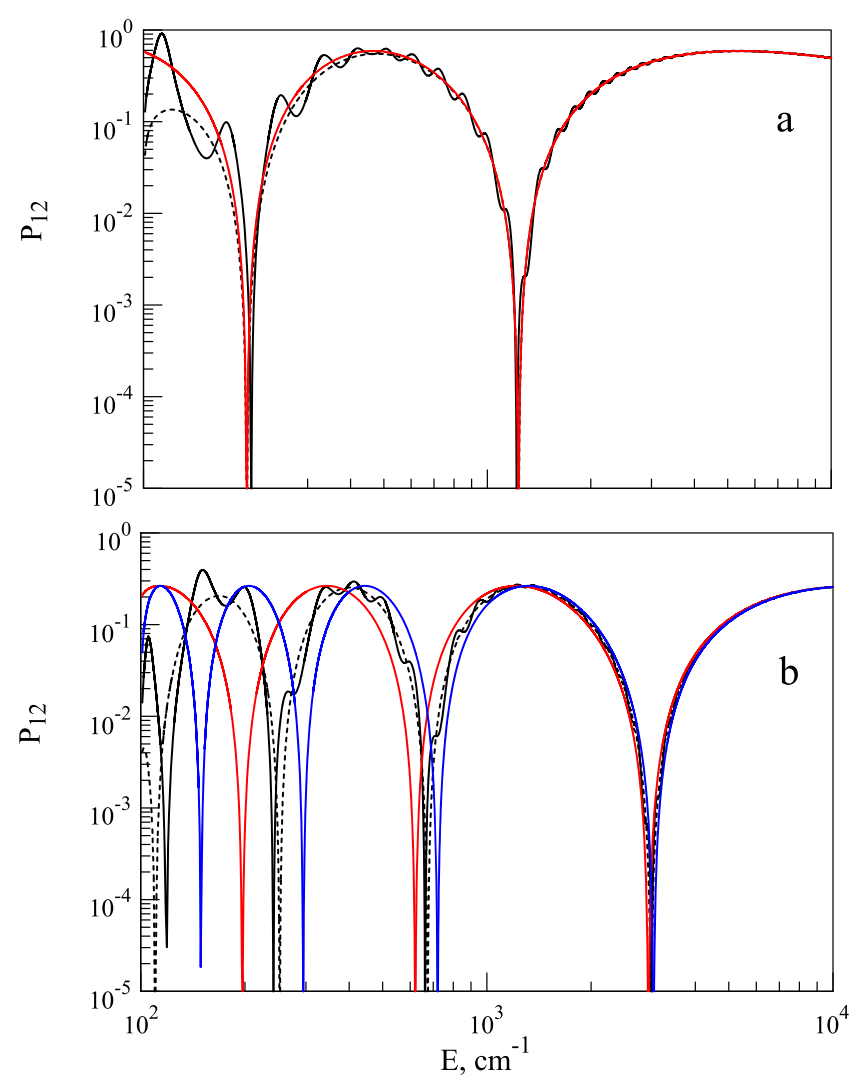

FIG. 3. Same as in Fig. 2, but the mixed quantum/classical approach is implemented using the average velocity method of Billing. Upper frame demonstrates excellent agreement for system 1 with identical potentials in the ground and excited states. Lower frame demonstrates poor agreement for system 2 with a large difference between the two potentials.

takes care of large $\Delta E$ ) because the entrance potential energy surfaces for excitation, $V_{11}$, and for quenching, $V_{22}$, are very similar (as in our model system 0 and system 1 , where we have set $\Delta V=0$ ).

Let us check what happens when $V_{11}$ and $V_{22}$ are different, by shifting the excited state potential to $V_{22}=100 \mathrm{~cm}^{-1}$ and keeping all other parameters as in system 1 (call this system 2). For this case, results of the mixed quantum/classical method with the average velocity approach of Billing already implemented (just as in the example above, as initial conditions) are shown in Fig. 3(b), together with two sets of quantum results (same line styles and colors). Now we see that two results of the mixed quantum/classical method (for excitation and quenching), again, deviate significantly from each other and in opposite directions from the quantum result. The Billing correction does not help anymore. The reason for this is that although the average velocity is set up at the initial moment of time, its value changes as the trajectory enters the interaction region, and does it differently for trajectories that start in the ground and excited potential energy surfaces. Only in the high energy limit, when the potential energy becomes less important, the mixed quantum/classical result is accurate [see Fig. 3(b)]. Interestingly, all anti-resonances are still there [four in this system, see Fig. 3(b)], but their positions are significantly shifted, leading to large errors, and more so at low energy. 
We have to admit that in our past MQCT calculations, we saw that, although the average velocity method of Billing worked for most cases, there were few transitions in couple molecules where it did not help. Examples include the transition between $2_{02}$ and $2_{20}$ states of $\mathrm{H}_{2} \mathrm{O}^{6}$ and the excitation of states $6_{6}$ and $4_{0}$ in $\mathrm{CH}_{3}$ from its ground state. ${ }^{10}$ We went back to these molecules, checked and found that indeed the potential energy surfaces are rather different for the initial and final states involved into these transitions, as in system 2.

A solution to this problem is our improved formula for the effective velocity, Eq. (21) which, in the case of large $\Delta V$, does better job (than the original approach of Billing). The result is shown in Fig. 4(a), where we see a dramatic improvement of the mixed quantum/classical result for system 2 [relative to Fig. 3(b)]. In this case, the excitation and quenching results are again identical, so, only a red line is seen. Agreement with the full-quantum result is now excellent, through the entire range of energies.

The last case to consider is system 3, obtained (from system 2) by significantly raising the value of coupling, to reach $V_{12}=150 \mathrm{~cm}^{-1}$. This is a significant modification, which creates seven "anti-resonances" in the same energy range, as one can see from Fig. 4(b). In this system, some quantum oscillations of transition probability remain visible even at high energies. Still, the mixed quantum/classical method [with a simplified effective velocity formula, Eq. (21), already implemented] gives, on average, a reasonable description at

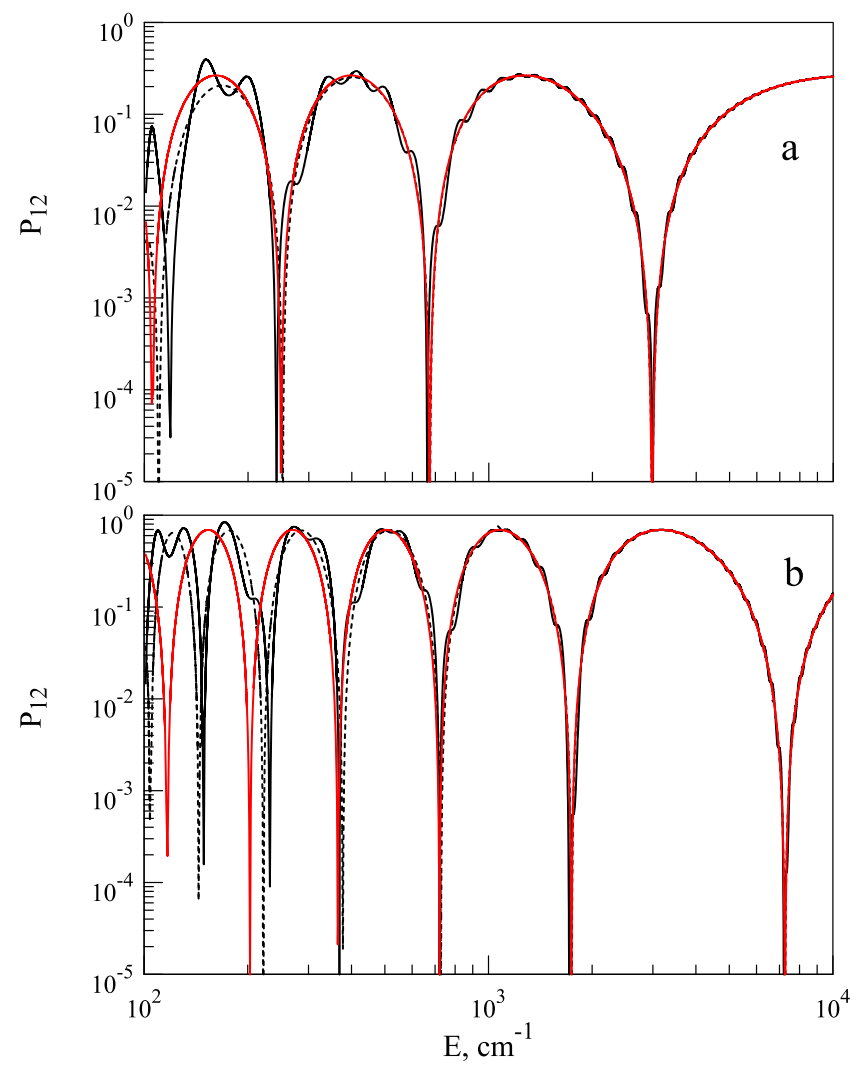

FIG. 4. Same as in Fig. 3, but the mixed quantum/classical approach is improved by using the effective velocity of Eq. (21). Upper frame, for system 2 with weak coupling between the ground and excited states, demonstrates excellent agreement with full-quantum results. Lower frame, for system 3 with strong coupling, demonstrates poor agreement.

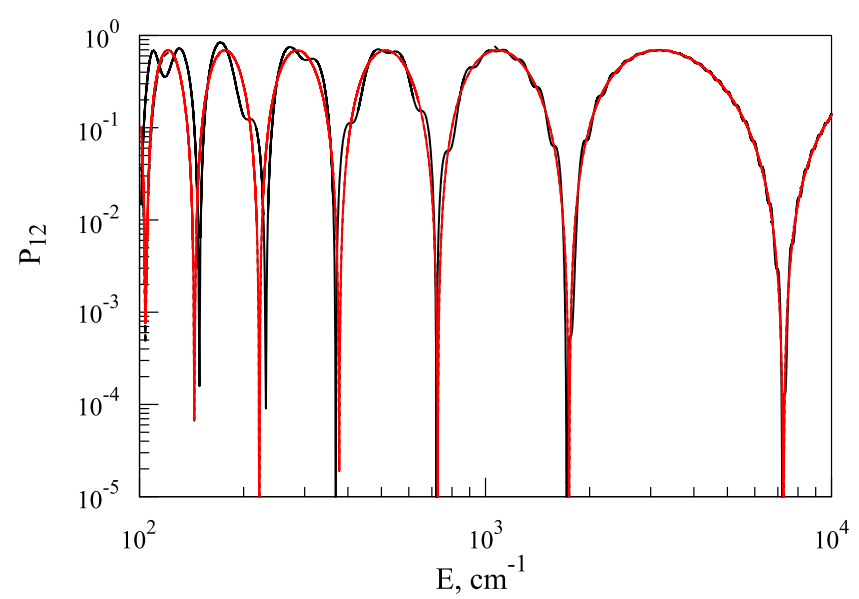

FIG. 5. Same as in Fig. 4, but the mixed quantum classical approach is implemented using the exact formula for the effective velocity, Eq. (20). Agreement with quantum results is excellent even for system 3 with strong coupling.

intermediate and higher energies. However, at low energies, it deviates from the full-quantum results. Even the approximate effective velocity of Eq. (21) is insufficient.

One solution to this problem is to use the most general form of the effective velocity, namely, the exact (although not particularly transparent, and somewhat lengthy) formula of Eq. (20). With this effective velocity, the mixed quantum/classical result matches almost exactly the prediction of an approximate quantum formula, Eq. (11). This is illustrated in Fig. 5, where solid red and dashed black lines are hardly distinguishable. Comparing Fig. 5 with Fig. 4(b), we see that the improvement due to Eq. (20) is very significant.

Such a case of strong coupling can be found in CO $+\mathrm{CO} \cdot{ }^{43}$ Preliminary calculations for this system (unpublished) revealed poor agreement between MQCT and the full quantum results, even if the average velocity of Billing is used. This system is considered next.

\section{APPLICATION TO REAL MOLECULES}

Four sets of model parameters considered in Sec. III help to understand three possible sources of errors in the mixed quantum/classical treatment of inelastic scattering. They occur due to large values of $\Delta E, \Delta V$, and $V_{12}$. In a given real molecule, only one of these may play a role or all three may happen to be relevant at the same time. In principle, the exact formula for effective velocity, Eq. (20), should help in either case. But the excellent accuracy we saw in Sec. III is guaranteed only for a simple model depicted in Fig. 1. In particular, the blue and red lines in Figs. 4 and 5 coincide completely only because in the model of Fig. 1 the velocity of motion through the interaction region does not depend on $V_{12}$ (as it was demonstrated and discussed in Sec. II). This is not necessarily the case in general, and some differences could occur between the mixed quantum/classical calculations of excitation and quenching in real molecules, even if Eq. (20) is used. This should also be checked by calculations.

But, it is not yet clear how to employ Eq. (20) in the multi-dimensional scattering calculations for real molecules, where the values of $V_{11}(x), V_{22}(x)$, and $V_{12}(x)$ are all smooth 
and continuously changing functions. One simple approach would be to use Eq. (20) with some representative values of $V_{11}, V_{22}$, and $V_{12}$, for example, the values typical to the interaction region (e.g., near the minimum, or near the turning point), where the state-to-state transitions are most intense. In a sense, this would be opposite to the prescription of Billing that is also specified for one single point on the trajectory (namely, for its initial point). Enforcing effective velocity in the interaction region is expected to work better, since most transitions occur in its vicinity, but it should somehow be taken into account that the scattering process is not one-dimensional (not as in the model of Fig. 1). Indeed, only a head-on moleculemolecule collision (the one with orbital angular momentum $l$ $=0$ ) is one-dimensional, but, its contribution to the cross section is typically small, as one can see from Fig. 6. Majority of important trajectories correspond to $l>0$. They experience centrifugal potential and, depending on the value of $l$, explore different parts of the potential surfaces $V_{11}(x)$ and $V_{22}(x)$, and experience a different amount of coupling $V_{12}(x)$. So, the estimation of the effective velocity must somehow be done for each individual trajectory, as a function of $l$, which would require a more involved algorithm of generating the initial conditions.

Another, completely different approach (not the meanfield trajectory per se) would be to "manually drag" the system along an artificial path such that it allows satisfying the average velocity condition at every moment of time. The following two-step procedure was implemented to try this idea. First, two elastic MQCT trajectories were propagated in a standard way, one for excitation and the other one for quenching, starting with the average velocity of Billing, that of Eq. (22). Along these trajectories, we recorded time-evolutions of classical variables (coordinates and momenta) and used them to construct an average path. At the second step, the equations for the time-evolution of probability amplitudes, analogous to those in Eq. (13), were integrated along this average path. This method of calculations is not proposed as a practical tool but rather as a demonstration of the main idea, of the principle, that could

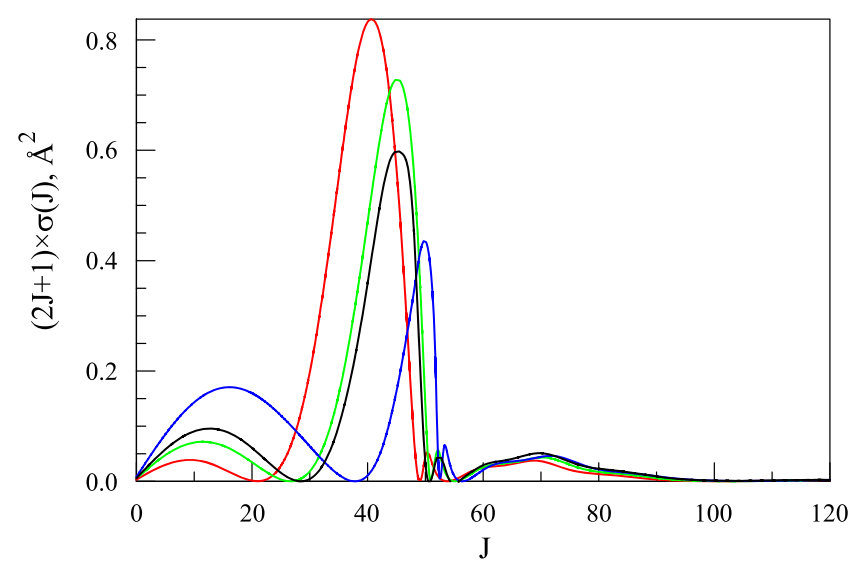

FIG. 6. Opacity function for excitation of the rotational state $j_{1} j_{2}(j m)=11(00)$ in $\mathrm{CO}+\mathrm{CO}$ collisions at total energy $E=100 \mathrm{~cm}^{-1}$. Black line is the exact quantum result, red and blue lines are MQCT results obtained from quenching and excitation calculations, with the average velocity method of Billing implemented. Green line is from the average path approach proposed in this work. be used for the development of an efficient algorithm in the future. Here, we will call it as the average path method, to differentiate it from the average velocity method of Billing that is specified only at the initial point. Technical note: Although one may think that the average trajectory is hard to define and may not even be unique, careful look at the MQCT equations formulated in the body-fixed reference frame ${ }^{3}$ reveals that all we need is to set, at every moment of time, the average value of the molecule-molecule separation and the average value of the orbital component of velocity (given by the time-derivative of azimuthal angle). Other classical variables are not required.

The tests are conducted for a real system, $\mathrm{CO}+\mathrm{CO}$, in which we only keep two internal states: the ground state $00(00)$ and one rotationally excited state $j_{1} j_{2}(\mathrm{jm})$. In this notation, $j_{1}$ and $j_{2}$ represent individual angular momenta of two molecules, while $j$ represent the total internal angular momentum of the system $\mathbf{j}=\mathbf{j}_{1}+\mathbf{j}_{2}$ (also quantized in MQCT ${ }^{8,9}$ ) and $m$ is its projection on the body-fixed reference frame. Note that $\mathbf{j}$ does not include the orbital angular momentum $\mathbf{l}$, since scattering is treated classically. Since initially $j=0$, the total angular momentum of the system $J=l$. Rotational constants for $\mathrm{CO}$ are $B_{e}=1.923 \mathrm{~cm}^{-1}$ and $D_{e}=6.12$ $\times 10^{-6} \mathrm{~cm}^{-1}$. Each $\mathrm{CO}$ is treated as a rigid rotor. Identical particle exchange symmetry is incorporated into MQCT. The potential energy surface for $\mathrm{CO}+\mathrm{CO}$ interaction is that by Dawes and co-workers. ${ }^{42,43}$

In the first example, the excited state is 11(00) at energy $E_{2}=7.69 \mathrm{~cm}^{-1}$ which corresponds to simultaneous excitations of both $\mathrm{CO}$ molecules into the first rotationally excited state. So, $\Delta E=7.69 \mathrm{~cm}^{-1}$ (relative to the ground state energy $E_{1}$ $=0$ ). The dependencies of $V_{11}(x), V_{22}(x)$, and $V_{12}(x)$ are given in the supplementary material. In the interaction region, the characteristic values are $V_{11}=59 \mathrm{~cm}^{-1}, V_{22}=39 \mathrm{~cm}^{-1}$, and $V_{12}=20 \mathrm{~cm}^{-1}$. This means that in this example, MQCT may suffer from both large $\Delta V$ and large $V_{12}$. Excitation energy $\Delta E$ is relatively small. The method of Billing, on its own, is not expected to be particularly useful.

In Fig. 6, we present the opacity function for this transition at relatively low total energy $E=100 \mathrm{~cm}^{-1}$. This gives a scaled transition probability or actually the excitation cross section, as a function of $J$. The full-quantum result (black solid line) computed using MOLSCAT ${ }^{44}$ serves as a reference. Two results of the standard MQCT, with the average velocity method of Billing already incorporated and carried out as excitation and quenching, are shown by red and blue lines, respectively. One interesting point, immediately obvious from Fig. 6, is that there is no simple way of averaging the red and blue lines (two MQCT results) to obtain the black line (full-quantum result) because these three curves appear to be shifted from each other along the $J$-axis. For example, near $J=26$, where blue and red curves cross (i.e., give the same value of cross section), the black curve is near zero. But, if one looks at the entire dependence, globally rather than locally, one observes that the evolution of the black line is, on average, "bracketed" by the red and blue lines. So, the mixed quantum-classical method caries accurate information about the scattering process. It would be nice to find a simple way of extracting it. 


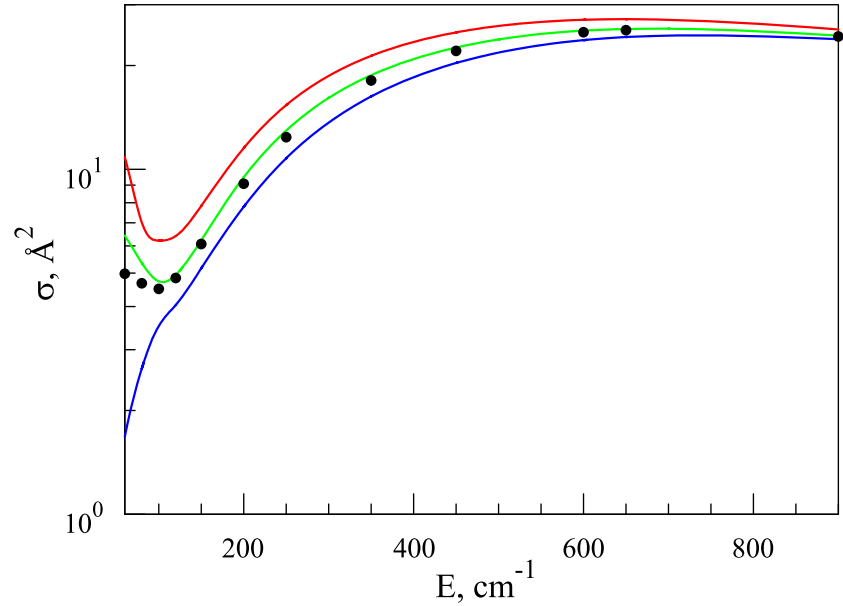

FIG. 7. Inelastic scattering cross section for excitation of state $j_{1} j_{2}(\mathrm{jm})$ $=11(00)$ in the $\mathrm{CO}+\mathrm{CO}$ system, starting from the ground state, as a function of total energy. Black symbols indicate the exact full-quantum data, while the green line is a result of the average path method. Red and blue lines are results of the MQCT calculation carried out as excitation (blue) or quenching (red), using the average velocity method of Billing.

Green line in Fig. 6 is the result of the average path method proposed above, and we see that it works much better. For all values of $J$ (and $l$ ), i.e., globally, the green line is closer to the full-quantum result than results of the other methods. So, enforcing reversibility at every point of trajectory (here by hands) works better than doing it at any one point, either the initial point (according to Billing) or in the interaction region (as we suggested above).

This message is further emphasized in Fig. 7, where the integral cross section for excitation of the rotational state $j_{1} j_{2}(\mathrm{jm})=11(00)$, starting from the ground state of $\mathrm{CO}+\mathrm{CO}$, is presented as a function of total energy. Again the pairs of red and blue lines (MQCT with average velocity of Billing) bracket black dots (the full quantum result). But our average path method (green line) works much better, giving an excellent description of the process at higher energies, and even a meaningful description at low energies.

In Fig. 8, the integral cross section for excitation of another rotational state, $j_{1} j_{2}(j m)=11(20)$, also starting from

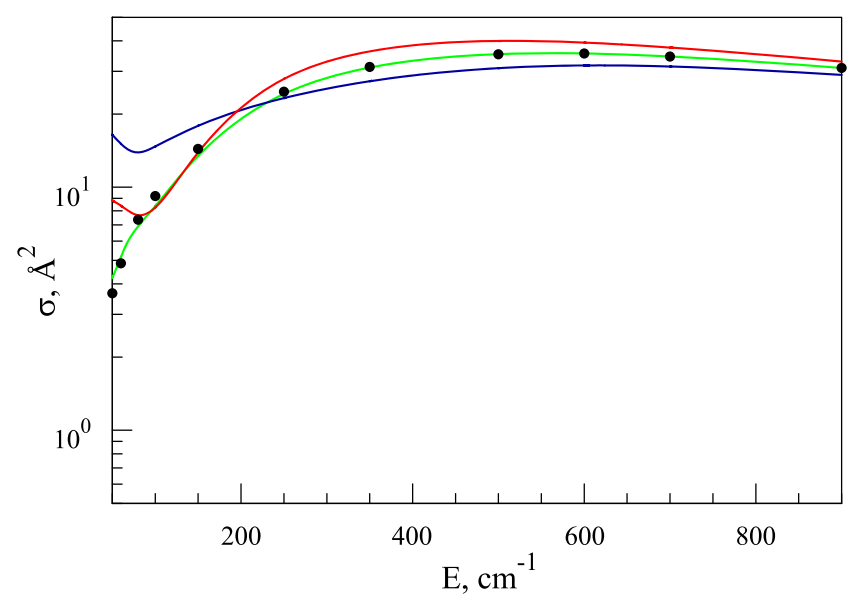

FIG. 8. Same as in Fig. 7, but for excitation of the rotational state $j_{1} j_{2}(\mathrm{jm})$ $=11(20)$ of the $\mathrm{CO}+\mathrm{CO}$ system. the ground state of $\mathrm{CO}+\mathrm{CO}$, is presented as a function of total energy. For this state, we also have $E_{2}=7.69 \mathrm{~cm}^{-1}$. The dependencies of $V_{11}(x), V_{22}(x)$, and $V_{12}(x)$ can be found in the supplementary material. In the interaction region, the characteristic values are $V_{22}=36 \mathrm{~cm}^{-1}$ and $V_{12}=30 \mathrm{~cm}^{-1}$ (i.e., rather similar to the first state we considered above). For this transition, we see that at intermediate and high energies, the results of MQCT with the average velocity of Billing (the pairs of red and blue lines) bracket the full quantum result (black dots) and monotonically converge to it in the limit of high energy. But at lower energies, predictions of MQCT become inaccurate. Interestingly, our average path method (green line) gives an excellent description of the process through the entire range of energies.

\section{CONCLUSION}

In order to identify the origin of errors in the mixed quantum/classical approach to inelastic scattering of molecules, we considered a simplified model that consists of one intermolecular degree of freedom and two intramolecular states, coupled by a simple potential. For this system, we derived analytically (i) an exact quantum mechanical solution of the inelastic scattering problem, (ii) a simplified version of it with all oscillation terms neglected, and (iii) a mixed/quantum classical solution in which the translational motion is described by the mean-field Ehrenfest trajectory, while the internal molecular motion is treated by the time-dependent Schrodinger equation. To our best knowledge, this has never been done before.

Analysis of these formulas reveals that the mixed quantum/classical description can produce nearly exact results if the velocity of the mean-field trajectory is appropriately chosen. If done correctly, this permits to obtain compatible results for the excitation and quenching processes, which also restores the microscopic reversibility, a known issue with the Ehrenfest approach. The average velocity method of Billing is rigorously derived as a limiting case of this approach, when reversibility is enforced locally, at the initial point of the Ehrenfest trajectory.

Further numerical experiments with these analytic expressions showed that deviations of the mixed quantum/classical results from the full-quantum results can occur at lower values of the total energy $E$ if the magnitudes of the excitation quantum $\Delta E$, the potential energy difference between two states $\Delta V$, and the coupling of two states $V_{12}$ are large. The effect of each of these three sources of errors is studied, separately, using four different sets of model parameters. It is shown that for some systems and collision conditions, very accurate results can be obtained by the mixed quantum/classical theory in its simplest form, some other cases may require setting up the initial conditions according to the average velocity recipe of Billing, but there may be cases when even this is not sufficient. For those cases, a new concept of effective velocity is proposed that can avoid problems due to large values of $\Delta E$, $\Delta V$, and $V_{12}$ (all of them at once, at least in the case of a simple model system considered in this work), by an appropriate choice of the initial conditions for the mixed quantum/classical trajectories. 
Possible ways of applying this concept to real molecular systems are explored, using examples from $\mathrm{CO}+\mathrm{CO}$ inelastic scattering on the actual potential energy surface. A practical algorithm for employing the effective velocity concept in the multi-dimensional MQCT calculations of inelastic scattering is yet to be found. An alternative ad hoc method is tried, in which the system is manually dragged along the average path, and is found to give results in excellent agreement with full quantum results. This exploratory implementation indicates new opportunities and may help to develop a numerically efficient computational tool for the description of inelastic scattering.

\section{SUPPLEMENTARY MATERIAL}

See supplementary material for the detailed derivation of analysis of equations and the potential energy surface data of $\mathrm{CO}+\mathrm{CO}$.

\section{ACKNOWLEDGMENTS}

This research was supported by NASA Astrophysics Program, Grant No. NNX17AH16G. We used resources of the National Energy Research Scientific Computing Center, which is supported by the Office of Science of the U.S. Department of Energy under Contract No. DE-AC02-5CH11231. Alexander Semenov acknowledges support of the Jobling Research Assistantship at Marquette.

$$
\left(\begin{array}{l}
A_{+} \\
A_{-} \\
C_{1} \\
C_{2}
\end{array}\right)=\left(\begin{array}{cc}
\sin a \kappa_{+} & \sin a \kappa_{-} \\
p_{+} \sin a \kappa_{+} & p_{-} \sin a \kappa_{-} \\
\kappa_{+} \cos a \kappa_{+} & \kappa_{-} \cos a \kappa_{-} \\
p_{+} \kappa_{+} \cos a \kappa_{+} & p_{-} \kappa_{-} \cos a \kappa_{-}
\end{array}\right.
$$

All elements of the inverted matrix can be obtained analytically, as shown in the supplementary material. But, among these four coefficients, we only need $C_{2}$, given by Eq. (9) in Sec. II A of the main text. From that, the element of the scattering matrix is

$$
\begin{aligned}
S_{12}=\sqrt{\frac{k_{2}}{k_{1}}} C_{2}= & -2 e^{-i a\left(k_{2}+k_{1}\right)} i \sqrt{k_{1} k_{2}}\left[\kappa_{+} \cos a \kappa_{+} \sin a \kappa_{-}\right. \\
& \left.-\kappa_{-} \cos a \kappa_{-} \sin a \kappa_{+}\right] /\left[p _ { + } \left(\kappa_{+} \cos a \kappa_{+}\right.\right. \\
& \left.-i k_{2} \sin a \kappa_{+}\right)\left(\kappa_{-} \cos a \kappa_{-}-i k_{1} \sin a \kappa_{-}\right) \\
& -p_{-}\left(\kappa_{+} \cos a \kappa_{+}-i k_{1} \sin a \kappa_{+}\right)\left(\kappa_{-} \cos a \kappa_{-}\right. \\
& \left.\left.-i k_{2} \sin a \kappa_{-}\right)\right] .
\end{aligned}
$$

One can formally obtain $S_{21}$ by switching indexes in this expression and then check that indeed $S_{21}=S_{12}$. A square of this matrix element gives a transition probability, Eq. (10), in Sec. II A of the main text. The expression in the brackets in the numerator of Eq. (10) can be rewritten, using trigonometric identities, as follows:

\section{APPENDIX: QUANTUM SOLUTION FOR THE TWO-STATE MODEL PROBLEM AND ITS PROPERTIES}

Matching Eq. (2) with Eq. (8) at $x=a$ (the boundary of interaction region) leads to the following four conditions:

$$
\left\{\begin{array}{l}
A_{+} \sin \kappa_{+} a+A_{-} \sin \kappa_{-} a=-e^{-i k_{1} a}+C_{1} e^{i k_{1} a} \\
A_{+} p_{+} \sin \kappa_{+} a+A_{-} p_{-} \sin \kappa_{-} a=C_{2} e^{i k_{2} a} \\
A_{+} \kappa_{+} \cos \kappa_{+} a+A_{-} \kappa_{-} \cos \kappa_{-} a=i k_{1} e^{-i k_{1} a}+C_{1} i k_{1} e^{i k_{1} a} \\
A_{+} p_{+} \kappa_{+} \cos \kappa_{+} a+A_{-} p_{-} \kappa_{-} \cos \kappa_{-} a=C_{2} i k_{2} e^{i k_{2} a}
\end{array} .\right.
$$

This system of linear equation can be solved in a standard way, by writing it in a matrix form

$$
\begin{gathered}
\left(\begin{array}{cccc}
\sin a \kappa_{+} & \sin a \kappa_{-} & -e^{i k_{1} a} & 0 \\
p_{+} \sin a \kappa_{+} & p_{-} \sin a \kappa_{-} & 0 & -i k_{2} a \\
\kappa_{+} \cos a \kappa_{+} & \kappa_{-} \cos a \kappa_{-} & -i k_{1} e^{i k_{1} a} & 0 \\
p_{+} \kappa_{+} \cos a \kappa_{+} & p_{-} \kappa_{-} \cos a \kappa_{-} & 0 & -i k_{2} e^{i k_{2} a}
\end{array}\right)\left(\begin{array}{c}
A_{+} \\
A_{-} \\
C_{1} \\
C_{2}
\end{array}\right) \\
=\left(\begin{array}{c}
-e^{-i k_{1} a} \\
0 \\
i k_{1} e^{-i k_{1} a} \\
0
\end{array}\right),
\end{gathered}
$$

and diagonalizing the matrix

$$
\left.\begin{array}{cc}
-e^{i k_{1} a} & 0 \\
0 & -e^{i k_{2} a} \\
-i k_{1} e^{i k_{1} a} & 0 \\
0 & -i k_{2} e^{i k_{2} a}
\end{array}\right)^{-1}\left(\begin{array}{c}
-e^{-i k_{1} a} \\
0 \\
i k_{1} e^{-i k_{1} a} \\
0
\end{array}\right)
$$

$$
\begin{aligned}
& \kappa_{+} \cos a \kappa_{+} \sin a \kappa_{-}-\kappa_{-} \cos a \kappa_{-} \sin a \kappa_{+} \\
& =\frac{\kappa_{+}-\kappa_{-}}{2} \sin a\left(\kappa_{+}+\kappa_{-}\right)-\frac{\kappa_{+}+\kappa_{-}}{2} \sin a\left(\kappa_{+}-\kappa_{-}\right) \\
& =\frac{a\left(\kappa_{+}^{2}-\kappa_{-}^{2}\right)}{2}\left(\frac{\sin a\left(\kappa_{+}+\kappa_{-}\right)}{a\left(\kappa_{+}+\kappa_{-}\right)}-\frac{\sin a\left(\kappa_{+}-\kappa_{-}\right)}{a\left(\kappa_{+}-\kappa_{-}\right)}\right) \\
& =\frac{2 m a V_{12}}{\hbar^{2}} \sqrt{R^{2}+1}\left(\frac{\sin a\left(\kappa_{+}+\kappa_{-}\right)}{a\left(\kappa_{+}+\kappa_{-}\right)}-\frac{\sin a\left(\kappa_{+}-\kappa_{-}\right)}{a\left(\kappa_{+}-\kappa_{-}\right)}\right) .
\end{aligned}
$$

Here we used the following formula:

$$
\frac{\hbar^{2}}{2 m}\left(\kappa_{+}^{2}-\kappa_{-}^{2}\right)=\frac{\left(\hbar \kappa_{+}\right)^{2}}{2 m}-\frac{\left(\hbar \kappa_{-}\right)^{2}}{2 m}=2 V_{12} \sqrt{R^{2}+1},
$$

obtained from Eqs. (5)-(7) in Sec. II A of the main text. Also, since $\left(\kappa_{+}^{2}-\kappa_{-}^{2}\right)=\left(\kappa_{+}-\kappa_{-}\right)\left(\kappa_{+}+\kappa_{-}\right)$, it gives two useful relations employed further below

$$
\frac{1}{\left(\kappa_{+}-\kappa_{-}\right)}=\frac{\hbar^{2}}{4 m V_{12}} \cdot \frac{\left(\kappa_{+}+\kappa_{-}\right)}{\sqrt{R^{2}+1}},
$$




$$
\left(\kappa_{+}-\kappa_{-}\right)^{2} \stackrel{E \rightarrow \infty}{\longrightarrow} \frac{2 m}{\hbar^{2}} \cdot \frac{V_{12}^{2}\left(R^{2}+1\right)}{E} .
$$

Using Eq. (A5), the numerator of Eq. (10) becomes

$$
16 \frac{k_{1} k_{2} m^{2} a^{2}}{\hbar^{4}} V_{12}^{2}\left(R^{2}+1\right)\left(\frac{\sin a\left(\kappa_{+}+\kappa_{-}\right)}{a\left(\kappa_{+}+\kappa_{-}\right)}-\frac{\sin a\left(\kappa_{+}-\kappa_{-}\right)}{a\left(\kappa_{+}-\kappa_{-}\right)}\right)^{2} .
$$

Manipulations with the denominator of Eq. (10) are somewhat lengthy and are presented separately in the supplementary material. The overall result for the modified form of Eq. (10) is

$$
\begin{aligned}
\left|S_{12}\right|^{2}= & 16 \frac{k_{1} k_{2} m^{2} a^{2}}{\hbar^{4}} V_{12}^{2}\left(R^{2}+1\right)\left(\frac{\sin a\left(\kappa_{+}+\kappa_{-}\right)}{a\left(\kappa_{+}+\kappa_{-}\right)}-\frac{\sin a\left(\kappa_{+}-\kappa_{-}\right)}{a\left(\kappa_{+}-\kappa_{-}\right)}\right)^{2} /\left[\frac{\kappa_{+}^{2} \kappa_{-}^{2}+k_{1}^{2} k_{2}^{2}}{2}\left(p_{+}-p_{-}\right)^{2}+\frac{\kappa_{+}^{2} \kappa_{-}^{2}-k_{1}^{2} k_{2}^{2}}{2}\left(p_{+}-p_{-}\right)^{2}\right. \\
& \times \cos a\left(\kappa_{+}+\kappa_{-}\right) \cos a\left(\kappa_{+}-\kappa_{-}\right)+\left(\frac{\left[p_{+}\left(k_{1} \kappa_{+}-k_{2} \kappa_{-}\right)+p_{-}\left(k_{1} \kappa_{-}-k_{2} \kappa_{+}\right)\right]^{2}}{4}-\frac{\left(\kappa_{+} \kappa_{-}-k_{1} k_{2}\right)^{2}}{4}\left(p_{+}-p_{-}\right)^{2}\right) \sin ^{2} a\left(\kappa_{+}-\kappa_{-}\right) \\
& +\left(\frac{\left[p_{+}\left(k_{1} \kappa_{+}+k_{2} \kappa_{-}\right)-p_{-}\left(k_{1} \kappa_{-}+k_{2} \kappa_{+}\right)\right]^{2}}{4}-\frac{\left(\kappa_{+} \kappa_{-}+k_{1} k_{2}\right)^{2}}{4}\left(p_{+}-p_{-}\right)^{2}\right) \sin ^{2} a\left(\kappa_{+}+\kappa_{-}\right) \\
& \left.+\frac{\left[p_{+}\left(k_{1} \kappa_{+}+k_{2} \kappa_{-}\right)-p_{-}\left(k_{1} \kappa_{-}+k_{2} \kappa_{+}\right)\right] \cdot\left[p_{+}\left(k_{1} \kappa_{+}-k_{2} \kappa_{-}\right)+p_{-}\left(k_{1} \kappa_{-}-k_{2} \kappa_{+}\right)\right]}{2} \sin a\left(\kappa_{-}-\kappa_{+}\right) \sin a\left(\kappa_{+}+\kappa_{-}\right)\right] .
\end{aligned}
$$

Although Eq. (A10) is exactly equivalent to Eq. (10) of the main text, this formula is easier to analyze. For such analysis, we should recall the definitions: $p_{ \pm}=R \mp \sqrt{R^{2}+1}$, where $R=(\Delta E-\Delta V) /\left(2 V_{12}\right)$ with $\Delta E=E_{2}-E_{1}$ and $\Delta V=V_{22}$ $-V_{11}$. This gives $\left(p_{+}-p_{-}\right)^{2}=4\left(R^{2}+1\right)$ and also $p_{+} p_{-}=-1$. One approximate version of Eq. (A10) is obtained by neglecting all oscillating terms. In the denominator, just the first term is retained, while in the numerator the second sine-function term is retained (see the supplementary material), which gives

$$
\begin{aligned}
\left|S_{12}\right|^{2} \approx & 16 \frac{k_{1} k_{2} m^{2} a^{2}}{\hbar^{4}} V_{12}^{2}\left(R^{2}+1\right) \frac{\sin ^{2} a\left(\kappa_{+}-\kappa_{-}\right)}{a^{2}\left(\kappa_{+}-\kappa_{-}\right)^{2}} \mid \\
& \times\left(\frac{\kappa_{+}^{2} \kappa_{-}^{2}+k_{1}^{2} k_{2}^{2}}{2}\left(p_{+}-p_{-}\right)^{2}\right) \\
= & \frac{8 m^{2}}{\hbar^{4}} V_{12}^{2} \frac{k_{1} k_{2} \sin ^{2} a\left(\kappa_{+}-\kappa_{-}\right)}{\left(k_{1}^{2} k_{2}^{2}+\kappa_{+}^{2} \kappa_{-}^{2}\right)\left(\kappa_{+}-\kappa_{-}\right)^{2}}
\end{aligned}
$$

Now we see, first of all, that if the coupling is small, $V_{12}$ $\rightarrow 0$, then the transition probability is also small, $\left|S_{12}\right|^{2} \rightarrow 0$, as expected. Using Eq. (A7), Eq. (A11) can be rewritten in a slightly different way, as it appears in the main text, Eq. (11).

We can also explore the high energy limit, $E \rightarrow \infty$. In this case, the following relations should be used: $\kappa_{+} \kappa_{-}$ $\rightarrow 2 m E / \hbar^{2}$ (which means that the values of kappa depend on energy only and not on the system properties) and $k_{1} k_{2}$ $\rightarrow 2 m E / \hbar^{2}$. Also, $\frac{\sin ^{2} a\left(\kappa_{-}-\kappa_{-}\right)}{a^{2}\left(\kappa_{+}-\kappa_{-}\right)^{2}} \rightarrow 1$ because $a\left(\kappa_{+}-\kappa_{-}\right) \rightarrow 0$ in the limit $E \rightarrow \infty$, due to Eq. (A8). For transition probability, this gives

$$
\left|S_{12}\right|^{2} \approx \frac{4 m^{2} a^{2}}{\hbar^{4}} V_{12}^{2} \frac{1}{k_{1} k_{2}}=\frac{2 m a^{2}}{\hbar^{2}} \cdot \frac{V_{12}^{2}}{E} .
$$

Another interesting property of Eqs. (A10) and (A11) is "anti-resonance," which is a possibility for transition probability to turn to zero at some value of energy. This happens when $\sin a\left(\kappa_{+}-\kappa_{-}\right)=0$ in the numerator of these equations or $\kappa_{+}-\kappa_{-}=\pi N / a$. Using Eq. (A8), which assumes relatively high energies (so, is used for qualitative analysis only), we obtain

$$
E=\frac{2 m a^{2}}{\pi^{2} \hbar^{2}} \frac{V_{12}^{2}\left(R^{2}+1\right)}{N^{2}}
$$

We see that positions (energies) of these "anti-resonances" depend on all parameters of the system and reversely proportional to $N^{2}$. The highest energy "anti-resonance" corresponds to $N=1$. The next is found at energy four times smaller, and the following one at energy nine times smaller, etc.

Finally, one can turn from excitation to quenching, which is the transition from state 2 to state 1 , assuming $E_{1}<E_{2}$, and look at the low-energy limit, as $E \rightarrow E_{2}$ (from above), $k_{2} \rightarrow 0$ (kinetic energy of collision is close to zero), and $k_{1} \approx \sqrt{2 m \Delta E} / \hbar$. This is done by using $\left|S_{21}\right|^{2}=\left|S_{12}\right|^{2}$ and $\sigma_{21}$ $=\frac{\pi}{k_{2}^{2}}\left|S_{21}\right|^{2}$. In this case,

$$
\frac{\left(\hbar \kappa_{ \pm}\right)^{2}}{2 m} \approx \frac{V_{22}+V_{11}}{2} \pm V_{12} \sqrt{R^{2}+1}
$$

So, the values of $\kappa_{+}$and $\kappa_{-}$depend on parameters of the system only (and not on collision energy). Then, we can simplify the equation as follows:

$$
\begin{aligned}
\left|S_{12}\right|^{2} & \approx \frac{8 m^{2}}{\hbar^{4}} k_{1} k_{2} \frac{V_{12}^{2} \sin ^{2} a\left(\kappa_{+}-\kappa_{-}\right)}{\kappa_{+}^{2} \kappa_{-}^{2}\left(\kappa_{+}-\kappa_{-}\right)^{2}} \\
& =\frac{8 m^{2}}{\hbar^{4}} k_{2} \frac{\sqrt{2 m \Delta E}}{\hbar} \cdot \frac{V_{12}^{2} \sin ^{2} a\left(\kappa_{+}-\kappa_{-}\right)}{\kappa_{+}^{2} \kappa_{-}^{2}\left(\kappa_{+}-\kappa_{-}\right)^{2}}, \\
\sigma_{21} & =\frac{\pi}{k_{2}^{2}}\left|S_{21}\right|^{2}=\frac{8 \pi m^{2}}{\hbar^{4}} \frac{\sqrt{2 m \Delta E}}{k_{2} \hbar} \cdot \frac{V_{12}^{2} \sin ^{2} a\left(\kappa_{+}-\kappa_{-}\right)}{\kappa_{+}^{2} \kappa_{-}^{2}\left(\kappa_{+}-\kappa_{-}\right)^{2}} .
\end{aligned}
$$


We see that quenching cross in the low-energy limit is reversely proportional to $k_{2}$, consistent with Wigner's law. The value of cross section depends on parameters of the system: $\Delta E, V_{12}$, $V_{11}$, and $V_{22}$, according to Eq. (A13).

${ }^{1}$ A. Semenov and D. Babikov, J. Chem. Phys. 138, 164110 (2013).

${ }^{2}$ A. Semenov, M. Ivanov, and D. Babikov, J. Chem. Phys. 139, 074306 (2013).

${ }^{3}$ A. Semenov and D. Babikov, J. Chem. Phys. 139, 174108 (2013).

${ }^{4}$ A. Semenov and D. Babikov, J. Phys. Chem. Lett. 5(2), 278 (2014).

${ }^{5}$ A. Semenov and D. Babikov, J. Chem. Phys. 140, 044306 (2014).

${ }^{6}$ A. Semenov, M.-L. Dubernet, and D. Babikov, J. Chem. Phys. 141, 114304 (2014).

${ }^{7}$ A. Semenov and D. Babikov, J. Phys. Chem. Lett. 6(10), 1854-1858 (2015).

${ }^{8}$ A. Semenov and D. Babikov, J. Phys. Chem. A 119(50), 12329 (2015).

${ }^{9}$ A. Semenov and D. Babikov, J. Phys. Chem. A 120(22), 3861 (2016).

${ }^{10}$ D. Babikov and A. Semenov, J. Phys. Chem. A 120(3), 319 (2016).

${ }^{11}$ K. J. McCann and M. R. Flannery, Chem. Phys. Lett. 35, 124-130 (1975).

${ }^{12}$ K. J. McCann and M. R. Flannery, J. Chem. Phys. 63, 4695-4707 (1975).

${ }^{13}$ G. D. Billing, The Quantum-Classical Theory (Oxford University Press, 2002).

${ }^{14}$ G. D. Billing, Comp. Phys. Rep. 1, 239 (1984).

${ }^{15}$ N. Balakrishnan and G. D. Billing, J. Chem. Phys. 101(4), 2785 (1994).

${ }^{16}$ N. Balakrishnan and G. D. Billing, Chem. Phys. Lett. 233, 145-153 (1995).

${ }^{17}$ G. D. Billing, Int. Rev. Phys. Chem. 13, 309 (1994).

${ }^{18}$ C. Coletti and G. D. Billing, J. Chem. Phys. 113(24), 11101 (2000).

${ }^{19}$ M. Ivanov and D. Babikov, J. Chem. Phys. 134, 174308 (2011).

${ }^{20}$ M. Ivanov and D. Babikov, J. Chem. Phys. 136, 184304 (2012).

${ }^{21}$ M. Ivanov and D. Babikov, Proc. Natl. Acad. Sci. U. S. A. 110, 17708 (2013).

${ }^{22}$ G. S. Whittier and J. C. Light, J. Chem. Phys. 110, 4280 (1999).

${ }^{23}$ D. Babikov, F. Aguillon, M. Sizun, and V. Sidis, Phys. Rev. A 59, 330 (1999).
${ }^{24}$ F. Chen and A. McCoy, J. Phys. Chem. A 107(37), 7220-7229 (2003).

${ }^{25}$ C. Tully and R. Johnson, J. Chem. Phys. 117, 6556 (2002).

${ }^{26}$ V. Ermoshin, A. Kazansky, and V. Engel, Chem. Phys. Lett. 302, 20 (1999).

${ }^{27}$ F. Aguillon, M. Sizun, V. Sidis, G. D. Billing, and N. Markovic, J. Chem. Phys. 104(12), 4530-4543 (1996).

${ }^{28}$ M. Sizun, F. Aguillon, V. Sidis, V. Zenevich, G. D. Billing, and N. Markovic, Chem. Phys. 209, 327-353 (1996).

${ }^{29}$ J. C. Tully, Faraday Discuss. 110, 407-419 (1998).

${ }^{30}$ A. D. McLachlan, Mol. Phys. 8, 39 (1964).

${ }^{31}$ H. D. Meyer and W. H. Miller, J. Chem. Phys. 72, 2272 (1980).

${ }^{32}$ D. A. Micha, J. Chem. Phys. 78, 7138 (1983).

${ }^{33}$ Z. Kirson, R. B. Gerber, A. Nitzan, and M. A. Ratner, Surf. Sci. 137, 527 (1984).

${ }^{34}$ S. I. Sawada, A. Nitzan, and H. Metiu, Phys. Rev. B 32, 851 (1985).

${ }^{35}$ H. J. C. Berendsen and J. Mavri, J. Phys. Chem. 97, 13464 (1993).

${ }^{36}$ P. Bala, B. Lesyng, and J. A. McCammon, Chem. Phys. Lett. 219, 259 (1994).

${ }^{37}$ M. Head-Gordon and J. C. Tully, J. Chem. Phys. 103, 10137 (1995).

${ }^{38}$ B. M. Garraway and K. A. Suominen, Rep. Prog. Phys. 58, 365 (1995).

${ }^{39}$ C. Marti, L. Pacifici, A. Lagan, and C. Coletti, Chem. Phys. Lett. 674, 103-108 (2017).

${ }^{40}$ A. Semenov and D. Babikov, "MQCT. I. Inelastic scattering of two Asymmetric-Top rotors with application to $\mathrm{H}_{2} \mathrm{O}+\mathrm{H}_{2} \mathrm{O}$," J. Chem. Theory Comput. (published online).

${ }^{41}$ A. Semenov and D. Babikov, "MQCT. II. A user-Ready Program for calculations of inelastic scattering, including collisions of two Asymmetric-Top rotor molecules," Comp. Phys. Comm. (unpublished).

${ }^{42}$ R. Dawes, X.-G. Wang, and T. Carrington, Jr., J. Phys. Chem. A 117(32), 7612-7630 (2013).

${ }^{43}$ S. Ndengué, R. Dawes, and F. Gatti, J. Phys. Chem. A 119(28), 7712-7723 (2015).

${ }^{44}$ J. Hutson and S. Green, MOLSCAT Computer Code, Version 14, distributed by Collaborative Computational Project No. 6 of the Engineering and Physical Sciences Research Council, Swindon, U.K., 1994. 